\title{
Non-invasive diagnosis: non-alcoholic fatty liver disease and alcoholic liver disease
}

\author{
Jose Altamirano ${ }^{1}$, Qiaochu $\mathbf{Q i}^{2}$, Sabina Choudhry ${ }^{3}$, Mohamed Abdallah $^{4}$, Ashwani K. Singal ${ }^{5}$, \\ Abhinav Humar ${ }^{6}$, Ramón Bataller ${ }^{6,7}$, Amir Ali Borhani ${ }^{8}$, Andrés Duarte-Rojo ${ }^{6,7}$ \\ ${ }^{1}$ Department of Internal Medicine (Hepatology Section), Hospital Quironsalud Barcelona, Spain; ${ }^{2}$ Department of Medicine, University of \\ Pittsburgh, Pittsburgh, PA, USA; ${ }^{3}$ Department of Radiology, ${ }^{4}$ Department of Internal Medicine (Hepatology Section), University of South Dakota, \\ Vermillion, SD, USA; ${ }^{5}$ Department of Medicine, University of South Dakota Sanford School of Medicine, Avera McKennan University Hospital \\ Transplant Hepatology, Sioux Falls, SD, USA; ${ }^{6}$ Starzl Transplantation Institute, ${ }^{7}$ Division of Gastroenterology and Hepatology, ${ }^{8}$ Department of \\ Radiology, University of Pittsburgh, Pittsburgh, PA, USA \\ Contributions: (I) Conception and design: A Duarte-Rojo; (II) Administrative support: None; (III) Provision of study material or patients: None; (IV) \\ Collection and assembly of data: None; (V) Data analysis and interpretation: None; (VI) Manuscript writing: All authors; (VII) Final approval of \\ manuscript: All authors. \\ Correspondence to: Andres Duarte-Rojo, MD, PhD. Starzl Transplantation Institute and Center for Liver Disease, University of Pittsburgh Medical \\ Center, Kaufmann Medical Building, Suite 201, 3471 Fifth Avenue. Pittsburgh, PA 15213, USA. Email: duarterojoa@upmc.edu.
}

\begin{abstract}
Non-alcoholic fatty liver disease (NAFLD) and alcoholic liver disease (ALD) are becoming the leading causes of chronic liver disease worldwide, significantly impacting public health and healthcare cost. The development of fibrosis is the main factor leading to early mortality and morbidity in NAFLD and ALD. Thus, it is important to timely and reliably evaluate these diseases at early stages, when fibrosis is not advanced or when steatosis predominates. Liver biopsy has been the standard of reference for fibrosis and steatosis, however, its invasiveness precludes its widespread use. There is growing research on non-invasive methods for diagnosing and stratifying fibrosis and steatosis in NAFLD and ALD. This review presents clinical evidence on the use of non-invasive assessment of liver disease (blood-based and imaging-based) in patients with NALFD and ALD, and proposes algorithms incorporating these tests into their management.
\end{abstract}

Keywords: Non-invasive diagnosis; non-alcoholic fatty liver disease (NAFLD); alcoholic liver disease (ALD); steatosis; fibrosis

Received: 10 July 2019; Accepted: 30 October 2019; Published: 05 April 2020.

doi: $10.21037 /$ tgh.2019.11.14

View this article at: http://dx.doi.org/10.21037/tgh.2019.11.14

Gastroenterologists and hepatologists are witnessing a change in the epidemiology of chronic liver disease. As the burden of chronic viral hepatitis $\mathrm{B}$ and $\mathrm{C}$ abates following the discovery of potent antiviral therapies, the global epidemics of obesity and alcohol abuse have created a new era with non-alcoholic fatty liver disease (NAFLD) and alcoholic liver disease (ALD) becoming the leading causes of chronic liver disease worldwide. Nonalcoholic steatohepatitis (NASH) will soon represent the main indication for liver transplantation in the United States and it is the fastest growing cause of hepatocellular carcinoma (1-3), whereas ALD is responsible for almost half of cirrhosis-associated deaths and hospital admissions $(4,5)$. Moreover, NAFLD and ALD can coexist and negatively impact other liver diseases, making it difficult to estimate their independent effect on public health and healthcare cost $(6,7)$. The diagnosis of NASH mandates a liver biopsy, and the identification of early stages of ALD also benefits from one, yet, the risks and costs associated with a liver biopsy preclude its widespread use. Pain is almost universal, and there is bleeding in approximately 1 in 500 liver biopsies and death in 1 in 10,000, dissuading patients from undergoing the procedure (8). Moreover, the disproportionately increasing incidence of NAFLD 
and ALD calls for practical and cost-effective care and monitoring (9). Methods for non-invasive assessment of liver disease (NIALD) and studies in the field have exponentially appeared over the last three decades, offering a safe and practical approach to hepatic fibrosis and steatosis quantification $(10,11)$. In this review, we will present the evidence supporting the use of NIALD to facilitate the care of patients with NAFLD and ALD.

\section{General principles of non-invasive assessment of liver disease}

Histologically, the spectrum of NAFLD is similar to ALD with (I) simple steatosis or fatty liver presenting initially, when there is $\geq 5 \%$ of parenchymal involvement by fat; followed by (II) steatohepatitis, once lobular inflammation, hepatocyte ballooning, and/or Mallory-Denk bodies ensue; (III) steatohepatitis with liver fibrosis, once collagen is deposited in the hepatic parenchyma, which typically first occurs in a centrilobular or perisinusoidal ("chicken-wire fence") fashion, followed by portal fibrosis; (IV) cirrhosis, when the fibrosis entraps and isolates hepatocytes into nodules; and (V) advanced liver disease, when complications of portal hypertension and/or hepatocellular carcinoma occur. Liver biopsy is considered the standard of reference to stratify NAFLD and ALD (12). Of the various histological scoring systems, the Brunt/Kleiner system is the most commonly used to stage fibrosis (F0 for none, F1 for perisinusoidal or periportal, F2 or significant fibrosis, when both perisinusoidal and periportal fibrosis are present, $\mathrm{F} 3$ or advanced fibrosis for presence of bridging, and $\mathrm{F} 4$ or cirrhosis) and to grade steatosis (5-33\% for mild, $>33-66 \%$ for moderate, and $>66 \%$ for severe) in NAFLD. The NAFLD activity Score (NAS), gives points for steatosis (0-3), lobular inflammation (0-3), and hepatocellular ballooning (0-2) on a full range of 0 to 8 , where patients with a score $\geq 5$ are considered to have definite nonalcoholic steatohepatitis (NASH), whereas those with a score $\leq 2$ are considered not to have NASH $(13,14)$. Regarding ALD, the alcoholic hepatitis histological scoring system can be used to stage fibrosis and grade steatosis in any clinical presentation (i.e., not only alcoholic hepatitis). In this score, although grading is similar to the Brunt/ Kleiner system, staging lumps stages F0 and F1 into a single category (15).

The presence of fibrosis is the most important histological feature as it is the most strongly associated with progressive liver disease and both hepatic and non-hepatic poor clinical outcomes both in NAFLD and ALD $(16,17)$. As such, cross-sectional validation of NIALD biomarkers has been performed using liver biopsy as the reference. However, liver biopsy is not an ideal reference given its inherent limitations in terms of sampling error, which causes inaccurate fibrosis staging in up to $33 \%$ of cases, and inter-observer variability with disagreement in staging of fibrosis in up to $30 \%$ of cases. Furthermore, research studies on NIALD validation tend to limit biopsies to $15 \mathrm{~mm}$ in length specimens with at least 10 complete portal tracts, quality characteristics that are not frequently observed in clinical practice (10). Hence, quality of liver biopsy and its inherent limitations when staging fibrosis need to be taken into account when contrasting histological results to those of NIALD biomarkers. Prospective validation of NIALD against clinical outcomes is of great relevance as it directly estimates the biomarkers' ability to prognosticate adverse outcomes (e.g., decompensation, mortality). Although there is a need for development of more accurate NIALD biomarkers to assess fibrosis staging and other features of liver disease (i.e., steatosis, NASH, alcoholic hepatitis), multiple blood-based and imaging-based biomarkers have been developed and validated in NAFLD and ALD, both cross-sectionally against liver biopsy and longitudinally against clinical outcomes.

\section{Usefulness of NIALD in NAFLD}

Approximately $20 \%$ of NAFLD patients (i.e., those with fibrosis and/or NASH) are at risk for progression to cirrhosis and its complications, including liver-related mortality (18). Thus, identification of fibrosis and features of NASH including steatosis is essential to identify patients requiring specific treatment and/or closer monitoring due to associated risk for progressive liver disease.

\section{Blood-based biomarkers}

Assessment of fibrosis: blood-based biomarkers to assess fibrosis in NAFLD can be categorized into indirect biomarkers (using patient demographics, standard of care laboratory values on liver chemistry and platelet counts) and direct biomarkers (using specific laboratory tests measuring extracellular matrix deposition and turnover) $(19,20)$. The most frequently used biomarkers in clinical practice are summarized in Table 1, and their characteristics are explained below.

NAFLD Fibrosis Score (NFS) incorporates age, 


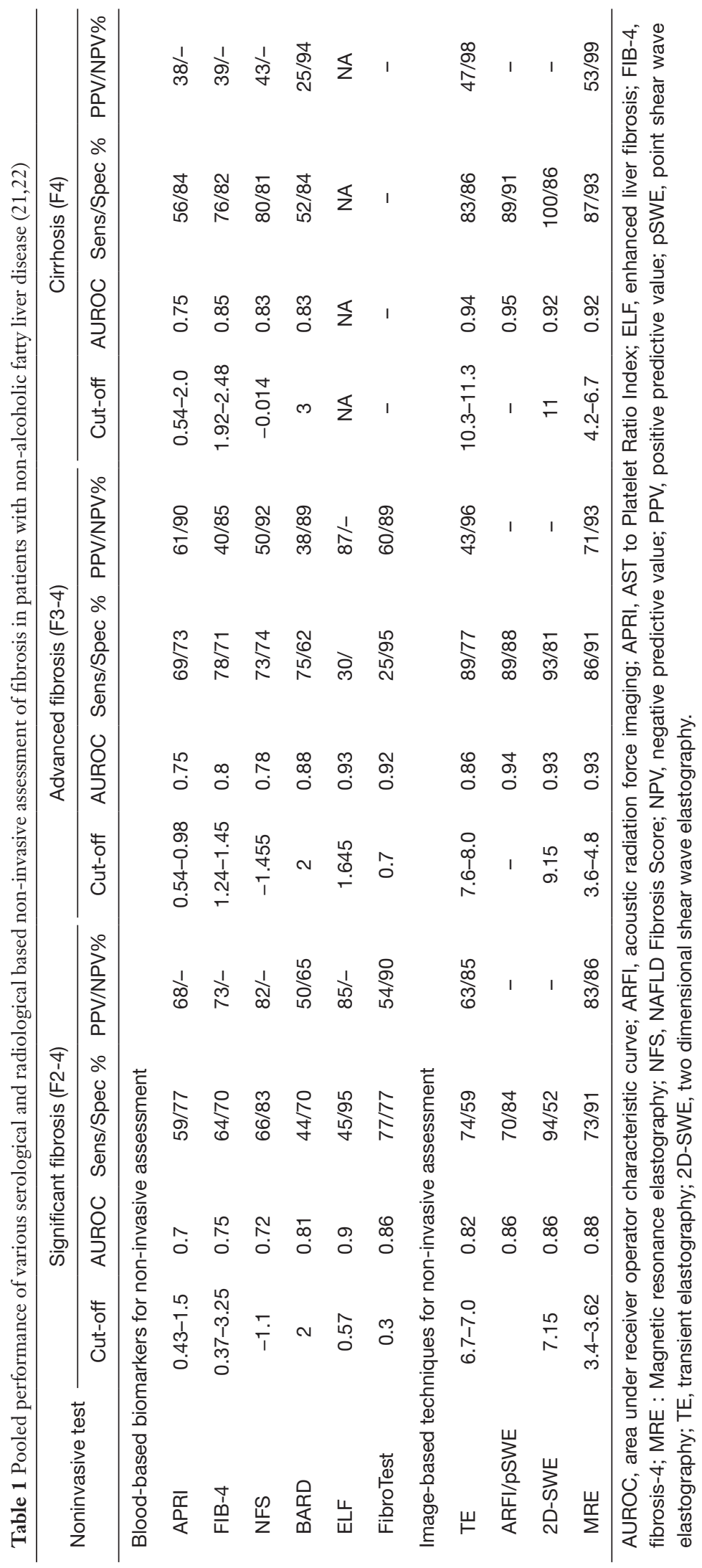


hyperglycemia, BMI, platelet count, serum albumin, and AST/ALT ratio, and in the pivotal study had AUC of 0.88 (0.85-0.92) and NPV of $93 \%$ to exclude F3-F4 fibrosis at NFS <-1.455 (23). As in many other NIALD biomarkers considering the platelet count (see below), the NFS is not accurate in patients with asplenia, or following placement of a transjugular intrahepatic portosystemic shunt (24). Similarly, NFS and other tests including AST and ALT lose accuracy when calculated during periods of acute-onchronic liver injury as aminotransferases might be acutely elevated.

Fibrosis-4 (FIB-4) uses age, AST, ALT and platelet count, and in the pivotal study on 832 biopsied patients with HIV and HCV coinfection, a cut off $>3.25$ had positive predictive value (PPV) of $68 \%$ and specificity of $97 \%$ in the diagnosis of advanced fibrosis (25). Based on the lower and upper cutoff of 1.30 and 3.25 , this test was able to avoid $71 \%$ of liver biopsies in this study. Later the test was found to be useful in NAFLD patients with an area under the receiver operating characteristics curve (AUROC) of 0.8 and 0.85 at respective cutoff values of $1.24-1.45$ and $1.92-2.48$ to make diagnosis of advanced fibrosis and cirrhosis respectively (Table 1).

AST to Platelet Ratio Index (APRI) was initially developed in hepatitis C patients, with AUROC (95\% CI) of 0.83 $(0.78-0.88)$ and 0.90 (95\% CI: 0.86-0.94) for predicting significant fibrosis and cirrhosis respectively (26). Utility of APRI among NAFLD patients was validated with respective AUROC of 0.77 and 0.91 at cut-off scores $0.43-1.5$ and 0.54-2.0 respectively (Table 1) (21).

$B A R D$ Score is the weighted sum of three variables (BMI $\geq 28=1$ point, AST/ALT ratio $\geq 0.8=2$ points, Diabetes $=1$ point). In the pivotal study, a score of $2-4$ among 827 NAFLD patients was associated with 17 -fold (9.2-31.9) odds of advanced fibrosis and was $96 \%$ accurate in excluding advanced fibrosis (27).

FibroTest ${ }^{\circledR}$ (FT) (or FibroSURE in the United States), uses total bilirubin, gamma glutamyl transferase (GGT), a2-macroglobulin, apolipoprotein A1, and haptoglobin, corrected for age and gender. In an initial NAFLD validation study, FibroTest showed an AUROC of 0.88 (95\% CI, 0.82-0.92) in predicting F3-4 fibrosis, proposing cutoff values of 0.3 and 0.7 to maximize NPV (90\%) and PPV (73\%), respectively (28). Importantly, conditions different from NAFLD affecting bilirubin (e.g., Gilbert's), haptoglobin (e.g., hemolysis), alpha-2-macroglobulin (e.g., inflammatory condition) or GGT (e.g., alcohol dinking), would result in a distorted and inaccurate estimation of fibrosis (10).

Enhanced liver fibrosis (ELF) is a score using direct biomarkers including matrix metalloproteinase 1 (tissue inhibitor of matrix metalloproteinase 1), hyaluronic acid, and amino terminal peptide of pro-collagen (P3NP) III, showing an AUROC of 0.90 for distinguishing F3-4 fibrosis (29). Caution is advised with the interpretation of ELF in patients having other diseases associated with increased collagen turnover (e.g., interstitial lung disease) (30) where a nonhepatic level of its components might be expected.

Comparative evaluation of serological markers in assessment of fibrosis in a meta-analysis of all the studies on over 13,000 patients (21), showed NFS and FIB-4 to be most accurate with pooled AUROC of 0.84 and negative predictive value (NPV) of $>90 \%$ for diagnosis of AF (Table 1). One of the limitation of the blood-based biomarkers is that approximately $20 \%$ to $30 \%$ of patients can fall into an indeterminate category, either below the low cutoff value used to rule out the condition being tested or above the high cutoff value to rule it in $(21,31)$. In these patients a liver biopsy would be recommended, although imaging-based NIALD can be used instead to further improve predictive accuracy and reduce the need for liver biopsy $(21,29)$.

Assessment of steatosis: many serum biomarkers have been developed to detect steatosis like the Fatty Liver Index, Hepatic Steatosis Index, SteatoTest, NAFLD Liver Fat Score, and index of NASH $(20,32,33)$. The accuracy of all these tests is comparable in the diagnosis of steatosis with AUROC between 0.80 to 0.83 (34). However, these are not often used in routine practice given accurate diagnosis of NAFLD using the recommended imaging criteria $(12,35)$.

\section{Imaging-based biomarkers}

Assessment of fibrosis: although multiple methods, mostly based on ultrasound and magnetic resonance technology, have been evaluated, we will restrict our analysis to the three most frequently validated (Table 1). In general, these methods are more accurate than their bloodbased counterparts, as recently shown in a head-to-head comparison against collagen proportionate area from liver biopsies (36), but they are less available and/or more costly in clinical practice.

Transient elastography (TE) or vibration controlled transient elastography is available as a point-of-care tool that is reproducible and easy to operate, and it provides objective staging data interpretable by hepatologists. As the test results can be affected by fed state, patients are advised to fast for at least 3 hours prior to the measurement (37). 

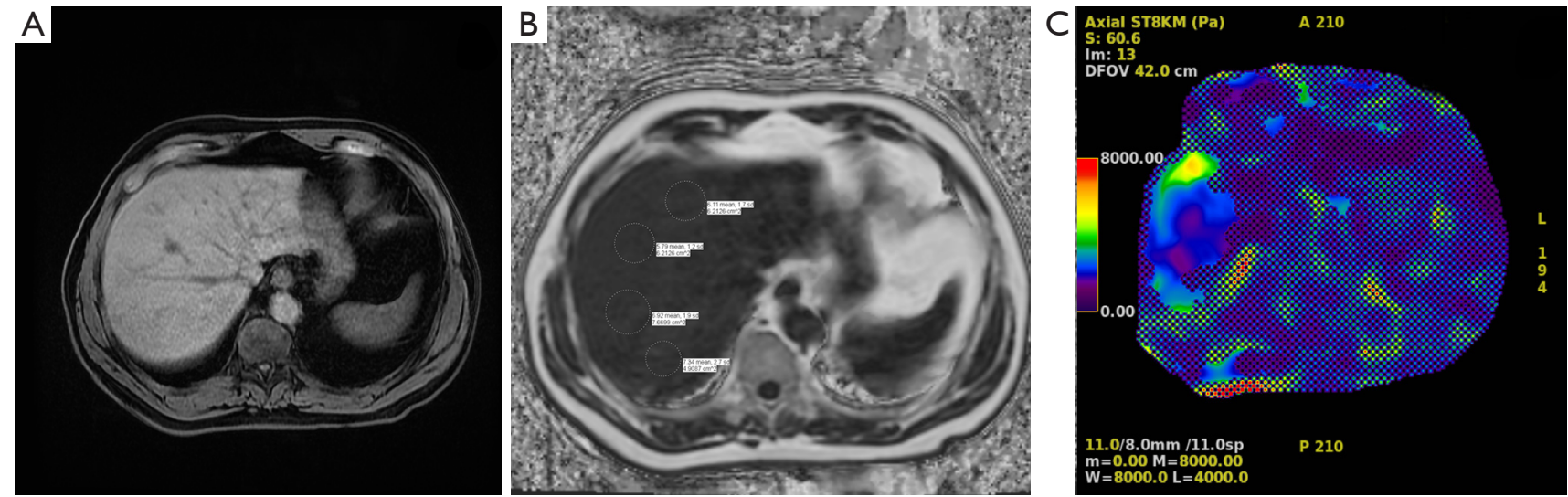

Figure 1 This is a case of a 66-year-old male with non-alcoholic fatty liver disease (NAFLD). (A) Unenhanced T1-weighted sequence showing the anatomy of the liver; (B) proton density fat fraction (PDFF), based on complex chemical shift encoded MRI (IDEAL IQ, GE Health), calculated to be $6.25 \%$ (Grade 0 steatosis); (C) MRE shows an average liver stiffness measure of $2.6 \mathrm{kPa}$ (F0/no fibrosis).

A skin-to-capsule distance $\geq 25 \mathrm{~mm}$, which is related to obesity and adiposity over the right upper quadrant area, precludes the use of the M-probe, and in such instances the $\mathrm{XL}$-probe is recommended. The test result (liver stiffness measurement or LSM) is reported in kilopascal $(\mathrm{kPa})$ units, with cut-off limits available to separate stages of fibrosis in NAFLD. TE is $94-100 \%$ accurate in ruling out F3-F4 at cut-off $8 \mathrm{kPa}$. The sensitivity and specificity for diagnosis of advanced fibrosis is $85 \%$ and $82 \%$ and for diagnosis of cirrhosis is $92 \%$ and $92 \%$ respectively (21). The AUROC for diagnosis of advanced fibrosis and cirrhosis does not differ comparing M- vs. XL-probe (0.87 vs. 0.86 and 0.92 vs. 0.94) (21), although some authors have reported lower cut-off values when using the XL-probe. TE combined with FIB-4 can improve the accuracy for diagnosis of advanced fibrosis or cirrhosis and reduce the need for liver biopsy, especially for patients when either of the two tests shows indeterminate results. However, the tool is limited by: lack of parenchymal assessment; inaccuracy in patients with ascites, morbid obesity, cholestasis, inflammation from acute hepatitis, and heart failure; operator and experience dependency (38). Considering the use of XL-probe for patients with a skin-to-capsule distance $\geq 25 \mathrm{~mm}$ (what is automatically prompted by the device to the operator), TE failure is observed in $<5 \%$ of patients with a body mass index $\geq 40 \mathrm{~kg} / \mathrm{m}^{2}$ (39).

Shear wave elastography (SWE): this can be performed using acoustic radiation force imaging (ARFI), also known as point shear wave elastography (pSWE) or two dimensional SWE (2D-SWE) (40). ARFI measures the ultrasound attenuation and velocity of spread of shear waves expressed as meter/second $(\mathrm{m} / \mathrm{s})$ and has shown an accuracy of $95 \%$ for the diagnosis of significant fibrosis $(22,41)$. 2D-SWE measures liver stiffness like TE in $\mathrm{kPa}$ units, with added advantage of assessment of hepatic parenchyma, and it has shown an accuracy of $92 \%$ for the diagnosis of advanced fibrosis in NAFLD (42). Both ARFI and 2D-SWE are becoming widely available, and in spite of the differences in cutoff values across manufacturers, high operator dependability, and limited experience (e.g., fewer prospective studies), accuracy is comparable to that of TE and represents a good alternative for NIALD in patients needing an anatomical ultrasound (11).

$M R$ elastography (MRE): mechanical waves generated in a drum device over the liver are imaged for about 15 seconds in end expiration, and the machine automatically provides a color-coded liver stiffness map (Figures 1-3). MRE has unique ability to assess regression, treatment response and progression on prospective follow up of patients during natural course or in response to treatment $(20,43)$. The accuracy of 2D-MRE for diagnosis of advanced fibrosis or cirrhosis has been documented in a recent metaanalysis (Table 1), and this is independent of BMI, gender, and degree of inflammation (44). Although 3D-MRE is more accurate than $2 \mathrm{D}$ technique, it takes more time and experience is limited (11). MRE has advantage over TE for better accuracy and of utility in morbidly obese and patients with ascites. However, this is limited by implanted metallic devices, claustrophobia, severe steatosis, hemochromatosis, cost, and lack of widespread availability. 

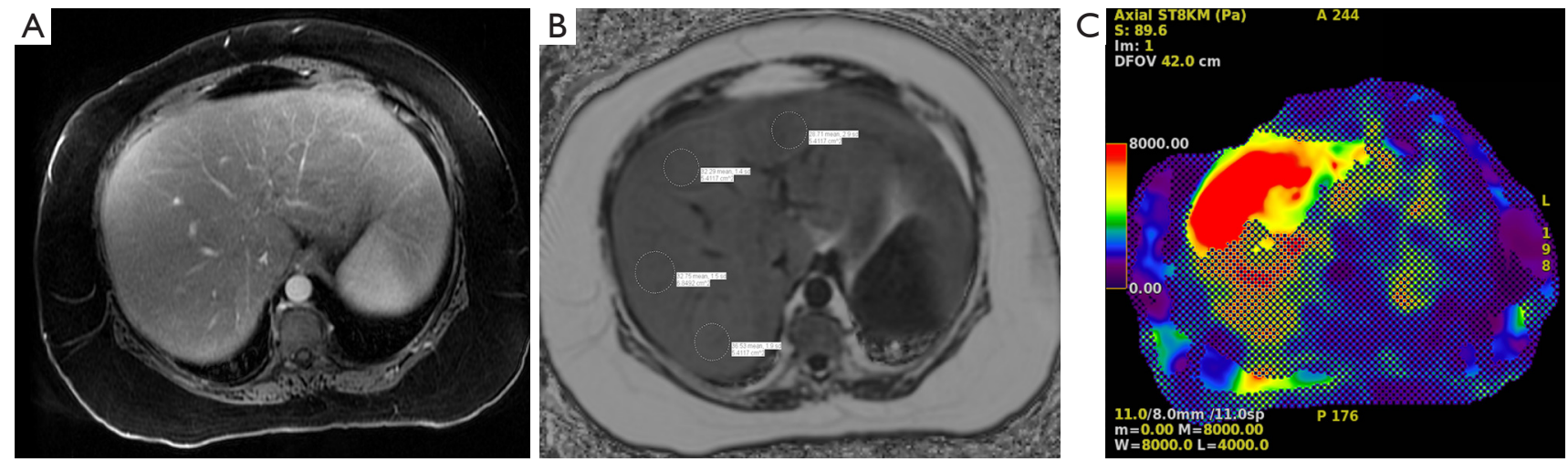

Figure 2 This is a case of a 53-year-old female with non-alcoholic steatohepatitis (NASH). (A) Venous-phase post-contrast T1-weighted MRI showing the anatomy of the liver; (B) proton density fat fraction (PDFF), based on complex chemical shift encoded MRI (IDEAL IQ, GE Health), was calculated to be 32.6\% (Grade 3 steatosis); (C) MRE of this patient with liver stiffness measured to be $5.7 \mathrm{kPa}$ (F4/ cirrhosis).
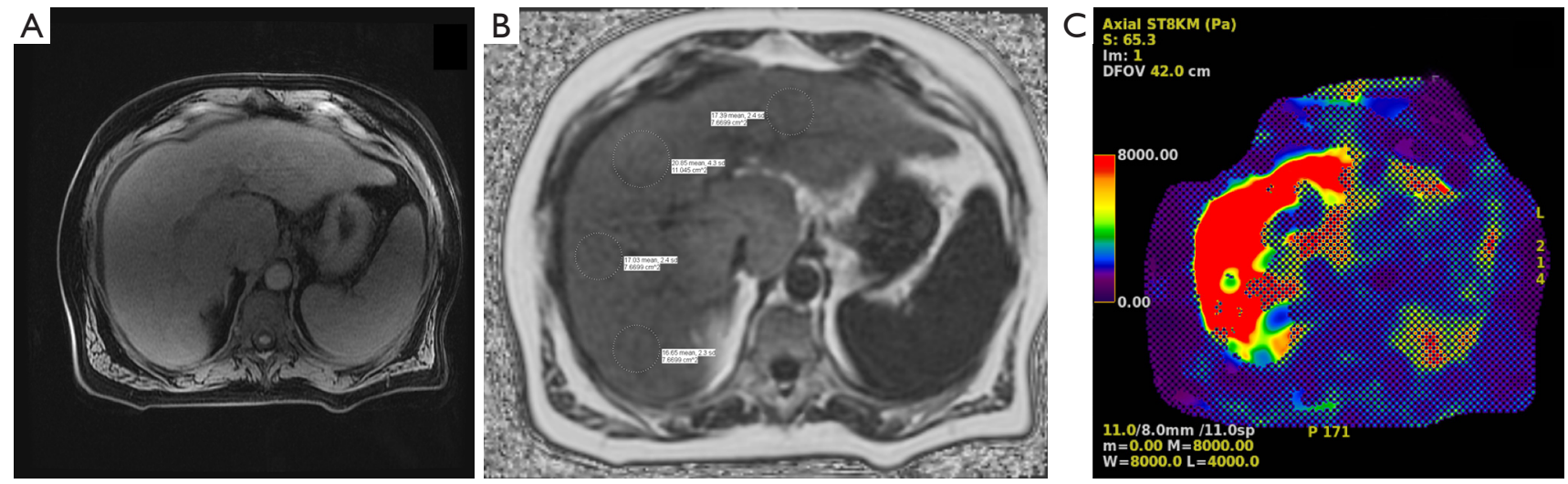

Figure 3 This is a case of a 61-year-old male with alcoholic hepatitis with cirrhosis. (A) Unenhanced T1-weighted sequence showing the anatomy of the liver; (B) MRI with Proton density fat fraction (PDFF), based on complex chemical shift encoded MRI (IDEAL IQ, GE Health), calculated to be $18 \%$ (Grade 2 steatosis); (C) MRE of this patient with liver stiffness measured to be $17.5 \mathrm{kPa}$ (F4/cirrhosis).

Assessment of steatosis: standard ultrasonography is the most widely available, inexpensive, well tolerated, and safe modality to diagnose and grade steatosis as mild (increased echogenicity compared to the right kidney), moderate (obliteration of the portal triads in the affected liver), and severe (attenuation of the hepatic parenchyma with nonvisualization of the right hemi diaphragm) (45). Despite ultrasonography high accuracy in the identification of parenchymal fat, subjectivity and operator dependence limit its usefulness for accurately grading steatosis. Further, the test can be falsely negative with steatosis of $6-10 \%$, morbid obesity, and concomitant renal disease $(46,47)$. In the absence of more available or widely validated method, ultrasonography is the method currently recommended for diagnosis of steatosis by both the AASLD and EASL $(12,35)$.

Controlled attenuation parameter (CAP) is a novel ultrasound-based technique able to quantify steatosis incorporated into the TE equipment. CAP processes the attenuation of ultrasound waves when travelling through fatty liver tissue with a proprietary algorithm, providing a numerical value that correlates with steatosis grading. It is easy to perform, widely available, accurate and reproducible. The pivotal study assessing this technique showed AUROC of 0.91, 0.95 and 0.89 for the diagnosis of mild, moderate, and severe steatosis (48). Since then, many studies have assessed this technique, however, they 


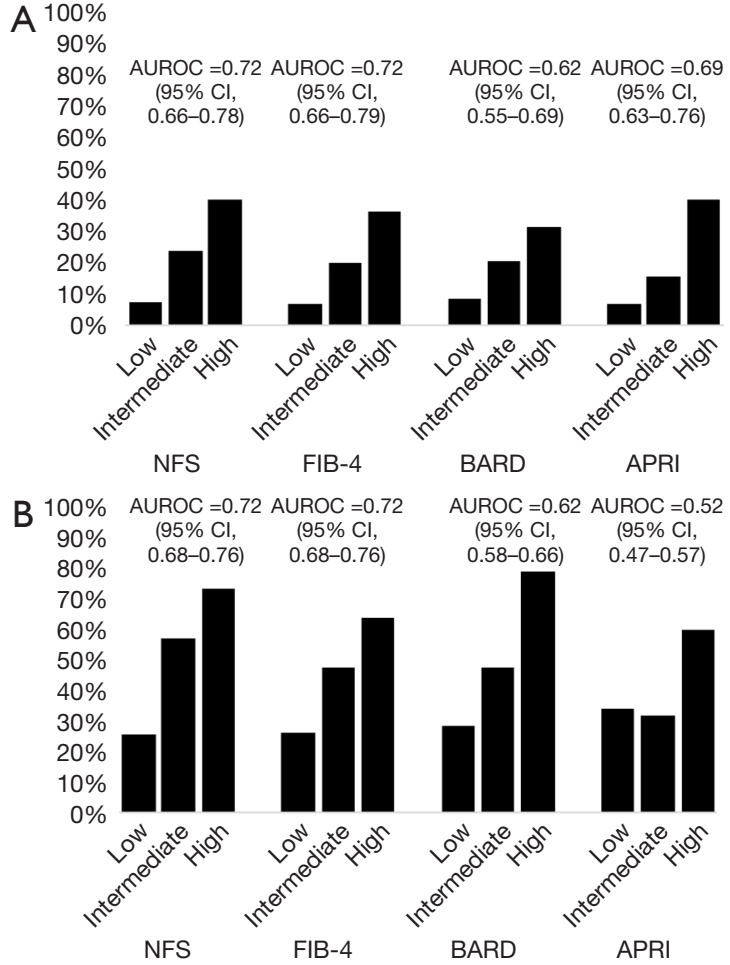

Figure 4 Percentages of study participants in Hagstrom et al.'s analysis who developed severe liver disease (A) and overall mortality (B) based on levels of scores for each of the four tests, along with AUROC for each test. AUROC: area under the receiver-operating characteristic curve. NFS, NAFLD fibrosis score; FIB-4, Fibrosis-4; BARD, BMI, AST/ALT ratio; APRI, aspartate aminotransferase-platelet ratio index.

are limited by small sample size. In a recent meta-analysis, these respective AUROC values have remained at $0.82,0.86$, and 0.88 at cut off 248,268 , and $280 \mathrm{~dB} / \mathrm{m}$ respectively (49). However, the tool is limited among patients with morbid or severe obesity and with ascites. In a limited evaluation of XL-probe $v s$. M probe, CAP has shown no difference in accuracy in quantifying fat (11). Some adjustments in CAP result are needed based on BMI and in those with NAFLD and diabetes (explained below).

MRI proton-density fat fraction (PDFF) depends on the ability of MRI technique to separate water and fat signals in any tissue including liver parenchyma, based on chemicalshift encoded MRI (Figures 1-3). MRI-PDFF is a reliable biomarker of hepatic steatosis, quantifying fat in the liver, and following patients on treatment for assessment of efficacy $(50,51)$. The technique is also superior to CAP for quantifying hepatic fat with AUROC of 0.99 vs. 0.85
$(\mathrm{P}=0.009)$ (52). Over the last decade, MRI-PDFF technique has also evolved to be accurate in measuring liver fat with excellent concordance to liver biopsy based quantification of liver fat at baseline as well as on follow up of patients (53). However, the technique is limited by lack of widespread availability, cost, expert needed for assessment, and other limitations of MRI based assessment.

\section{Prospective NAFLD studies using NIALD}

Apart from cross-sectional assessment, biomarkers predicting progressive disease or post-treatment changes are needed. In NAFLD, fibrosis stage is the most important liver biopsy parameter identifying progressive liver disease and predicting a higher risk of mortality and liver transplantation (16). Fibrosis is also associated with higher incidence of cardiovascular disease, metabolic syndrome, non-hepatic and hepatocellular malignancy $(17,54)$. Stratifying patients with NAFLD early on and following them regularly for re-stratification is important for predicting and managing complications from NAFLD. Thus, noninvasive imaging methods are valuable tools for this purpose. Blood-based biomarkers have also been shown to predict liver-related outcomes and overall mortality (Figure $4 A, B)(31,55,56)$. Also, higher stages of fibrosis per LSM from TE have been associated with increased mortality, and increased carotid arterial stiffness was found in patients with $>8.7 \mathrm{kPa}$ (M-probe)/7.2 kPa (XL-probe) (57). Similarly, increasing fibrosis staging from MRE is associated with increased coronary artery calcium in patients with type 2 diabetes mellitus (58).

\section{A practical approach on the use of NIALD for patients with NAFLD}

Considering the accuracy of NIALD techniques validated against liver biopsy, we have developed a clinical care algorithm to approach patients referred with a presumptive diagnosis of NAFLD (Figure 5). Out of the multiple available methods we favor the use of NFS or FIB-4 (among the most accurate blood-based methods with a particularly high NPV), as they can be calculated from history and routine blood work, and TE or MRI. Although MRE is the more accurate commercially available image-based method, TE allows point-of-care clinical decisions with a comparable accuracy when good quality elastograms (low failure and variability; mostly BMI $<40 \mathrm{~kg} / \mathrm{m}^{2}$ ) are obtained. As such, we aim to perform TE in all comers, except when 


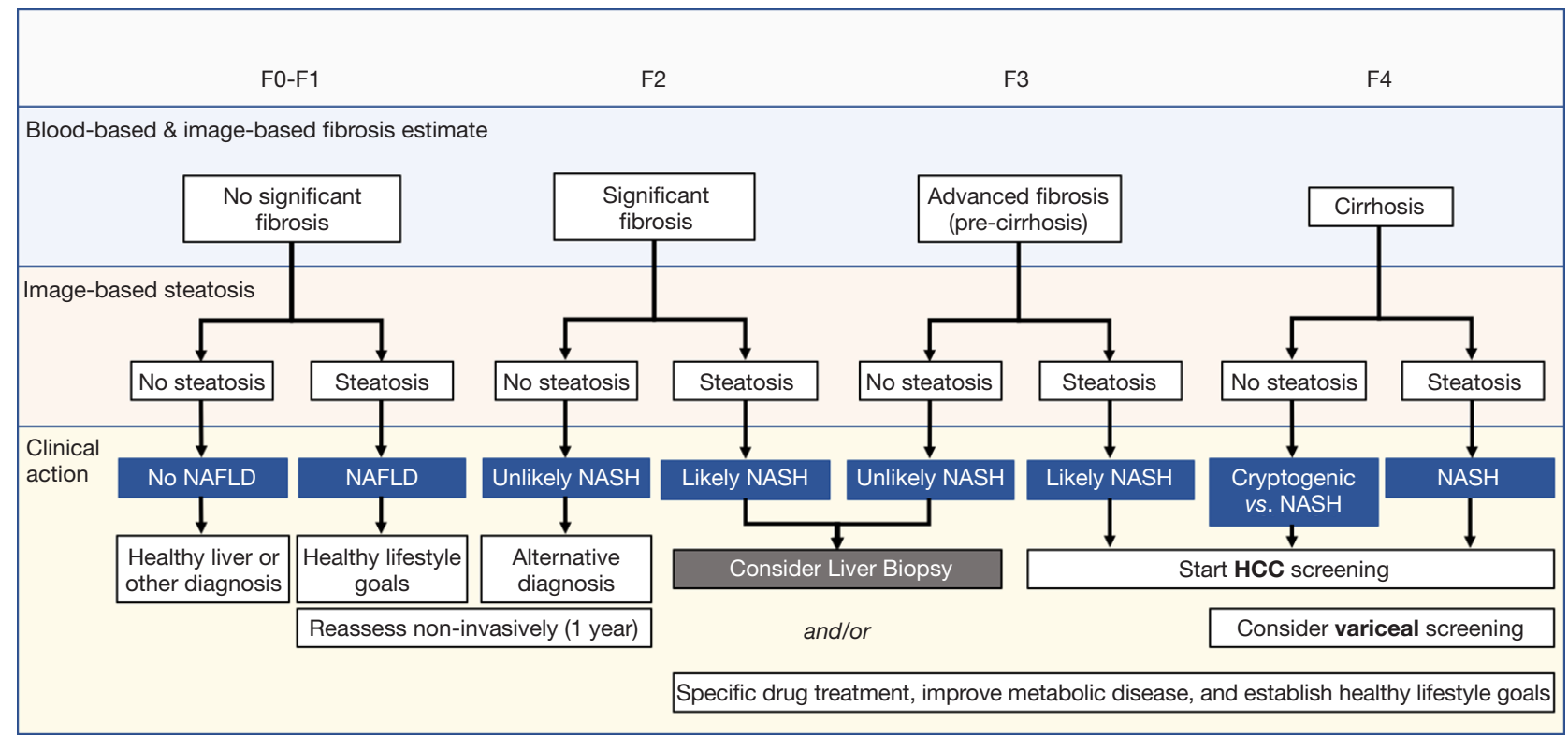

Figure 5 Proposed algorithm for fibrosis stratification, potential screening, and management in patients with non-alcoholic fatty liver disease (NAFLD). NASH, non-alcoholic steatohepatitis; HCC, hepatocellular carcinoma.

body frame or lack of insurance coverage precludes pointof-care testing. The use of two NIALD tests to better characterize NAFLD can be done in a simultaneous (paired) or sequential (serial) fashion. In the paired algorithm, agreement between the two tests is expected, increasing correct classification, whereas the serial approach employs a sensitive or specific test as first line, leaving the second test to reclassify patients with uncertain results ("grey zone" or non-classifiable). The latter decreases algorithm's failure rate and would result in less liver biopsies (59). We acknowledge that single testing (particularly MRE/PDFF) suffices for this algorithm in most cases and that a serial approach is more cost-efficient, however, we tend to base important clinical decisions on concordant test results (e.g., both FIB-4 and LSM agree on presence of F3). Discordant results are followed by a liver biopsy when clinically pertinent. Importantly, if a biopsy is not obtained, continued follow-up with repeated testing potentially decrease the risk of incorrect classification (i.e., failing to identify F0-1 or F2 NAFLD). We monitor our patients with NIALD testing on a yearly basis.

Fibrosis plays a central role in prognosticating NAFLD, whereas steatosis has an inverse association with fibrosis and thus its prognostic role is limited to early stages of the disease. However, steatosis is paramount for screening and diagnosing NAFLD and NASH at most stages, except for advanced cirrhosis. Thus, our algorithm starts with fibrosis classification and then adds CAP or PDFF to help identify NAFLD, under the assumption that other causes of hepatic steatosis have already been ruled out (e.g., alcohol abuse, medications, viral hepatitis, Wilson's disease, etc.). Patients with F0-1 or F2 on the basis of NIALD and no steatosis, likely do not have NAFLD, although yearly repeated testing might be pertinent in those with F2 (significant fibrosis), as well as in patients with $\mathrm{F} 0-1$ with steatosis. The presence of F2 with steatosis or F3 (advanced fibrosis) without steatosis calls for a liver biopsy, although in patients who are reluctant to undergo this procedure starting treatment is a reasonable option. This is particularly true if steatosis is at least moderate or if there are multiple components of the metabolic syndrome (e.g., obesity, diabetes mellitus, insulin resistance, and/or dyslipidemia). Few studies have aimed to validate LSM/CAP or MRE/PDFF to identify NASH, however, NIALD cut-off values and criteria for diagnosing NASH differed across them. When using LSM/CAP, the AUROC for diagnosing NASH is $0.35-0.81$ and with MRE/ PDFF it lies between $0.70-0.81$. With such a significant potential for missing a clinically-relevant diagnosis, based on these results, the consensus continues to be that NASH can solely be diagnosed by histopathology. Yet, research incorporating other imaging techniques and combining them with blood-based biomarkers is encouraging. Among 
these is cytokeratin-18 fragments (caspase-generated intermediate filament during apoptosis) which have shown an AUROC of 0.83 for the diagnosis of NASH (60). Finally, patients with $\mathrm{F} 3$ and steatosis or F4 (cirrhosis) should not be delayed for treatment and appropriate screening. Although still controversial, in selected cases we begin HCC screening in patients with F3 with 6-monthly ultrasound or another method when ultrasound quality is inadequate. Similarly, in the presence of an LSM $>20-25 \mathrm{kPa}$ (or MRE equivalent $\approx 8 \mathrm{kPa}$ ), we perform esophagogastroscopy for variceal screening, as per recommended guidelines. In NAFLD patients in need of a surgical procedure (liver resection or bariatric) a similar finding would preclude surgery due to the presence of clinically significant portal hypertension.

It is important to mention that some clinical variables need to be taken into account when applying this algorithm into practice. Body frame, BMI, obesity, diabetes, steatosis and race are known to affect NIALD, particularly the imaging-based methods (11). As an example, CAP readings need to be corrected by deducting $10 \mathrm{~dB} / \mathrm{m}$ in NALFD, 10 $\mathrm{dB} / \mathrm{m}$ in the presence of diabetes mellitus; and by deducting or adding 4.4 per BMI above or below $25 \mathrm{~kg} / \mathrm{m}^{2}$ (over the 20-30 range), respectively (49). Thus, in a patient with NAFLD, diabetes and a BMI of $30 \mathrm{~kg} / \mathrm{m}^{2}$, a measured CAP of $318 \mathrm{~dB} / \mathrm{m}$ is adjusted to $276 \mathrm{~dB} / \mathrm{m}$ (reclassifying steatosis from severe to moderate), whereas in a patient with lean NAFLD and a BMI of $22 \mathrm{~kg} / \mathrm{m}^{2}$ a CAP of 230 is adjusted 243 (reclassifying steatosis from absent to mild).

\section{Dynamic changes in NIALD following therapeutic strategies}

The recognition of NASH with fibrosis as a reversible entity following a lifestyle intervention, along with the development of antifibrotic and disease-specific therapies, has generated a demand for non-invasively monitoring changes in fibrosis and/or steatosis. Among the bloodbased biomarkers, FIB-4, APRI, and NFS are the ones more consistently paralleling regression or progression of fibrosis in studies utilizing paired biopsies (before and after intervention). A recent study combining the experience of two NIH-sponsored studies $(\mathrm{n}=292)$ identified AUROC of $0.82,0.81$, and 0.80 for APRI, FIB-4, and NFS for progression to advanced fibrosis, although none of them could detect regression of fibrosis. According to this study, a change in 1 stage of fibrosis (scale of F0 to F4) was associated with changes of 0.33 units of APRI, 0.26 of FIB-4, and 0.19 of NFS (61) after a median follow-up of
2.6 years. In a similar study including 261 non-cirrhotic patients, out of these three parameters, only NFS predicted progression or regression of fibrosis after 1 year, however, a novel index composed of change in hemoglobin A1c, platelet count, and ALT normalization (the FILI scoreFibrosis Improvement after Lifestyle Interventions) better predicted regressed fibrosis with PPV and NPV of $94 \%$ and $91 \%$, respectively (62). Given the strong interrelationship between the metabolic syndrome and NASH, the FILI score needs to be further validated following interventions not targeting weight loss and an improved metabolic profile. Also, since hemoglobin A1c loses accuracy in the setting of cirrhosis-especially if decompensated-it is less clear whether this index can be reproduced patients with $\mathrm{F} 4$ staging (63).

Imaging-based methods are also promising to detect changes in fibrosis in NAFLD. Among patients with baseline and follow-up biopsies treated with selonsertib (an apoptosis signaling inhibitor) for 24 weeks, MRE could not detect changes in fibrosis ( $\mathrm{n}=54$ ), although MRI-PDFF was sensitive to improvements in steatosis $(n=65)$ when compared to histological responders (64). Although not validated against paired liver biopsies, other studies have shown that MRE/MRI-PDFF or LSM/CAP improve in response to NASH treatment. In a randomized clinical trial testing an allosteric inhibitor of acetyl-Co A for 12 weeks ( $\mathrm{n}=126)$, a study showed reduction in MRI-PDFF $\geq 30 \%$ only in patients receiving this experimental drug at the highest dose, along with a trend for MRE reduction but no significant changes for LSM/CAP (65). A clinical trial evaluating the effect of 24 weeks of dapagliflozin in 57 NAFLD patients with diabetes mellitus showed an improvement in LSM (from $15 \pm 6$ to $11 \pm 7 \mathrm{kPa}$ in the group with significant fibrosis at baseline) and in CAP (from $314 \pm 61$ to $290 \pm 73 \mathrm{~dB} / \mathrm{m}$ ) (66). As such, although identifying regression/progression in staging or grading of NAFLD is a possibility with NIALD, further validation is needed including head-to-head comparisons between imagingbased and blood-based methods. It is possible that the inferior performance of imaging-based method relates to the short follow-up and absence of an effective and durable intervention in reported studies.

\section{Usefulness of NIALD in ALD}

Liver steatosis is considered the initial lesion of ALD and is present in approximately $90 \%$ of heavy drinkers, however, only $10-20 \%$ will eventually accumulate fibrosis and progress 
Table 2 Pooled performance of various serological and radiological based non-invasive assessment of fibrosis in patients with alcoholic liver disease

\begin{tabular}{|c|c|c|c|c|c|c|c|c|}
\hline \multirow{2}{*}{ Noninvasive test } & \multicolumn{4}{|c|}{ Significant Fibrosis (F2-4) } & \multicolumn{4}{|c|}{ Cirrhosis (F4) } \\
\hline & Cut-off & AUROC & Sens/Spec \% & PPV/NPV\% & Cut-off & AUROC & Sens/Spec \% & PPV/NPV\% \\
\hline \multicolumn{9}{|c|}{ Blood-based biomarkers for non-invasive assessment } \\
\hline PGAA & $>10$ & 0.83 & $54 / 98$ & $97 / 64$ & $>10$ & 0.87 & $80 / 88$ & $72 / 92$ \\
\hline FibroTest & & 0.85 & & & 0.58 & 0.88 & $67 / 87$ & $60 / 90$ \\
\hline \multicolumn{9}{|c|}{ Image-based techniques for non-invasive assessment } \\
\hline \multicolumn{9}{|l|}{ TE/fibroscan } \\
\hline 1. AST $<39 \mathrm{IU} / \mathrm{TB}<0.5 \mathrm{mg} / \mathrm{dL}$ & 6.9 & 0.87 & $80 / 77$ & $78 / 79$ & 12.1 & 0.92 & $85 / 84$ & $97 / 51$ \\
\hline $\begin{array}{l}\text { 2. AST } 39-75 \mathrm{IU} / \mathrm{TB}<0.5 \mathrm{mg} / \mathrm{dL} \text { or } \\
\text { AST }<39 \mathrm{IU} / \mathrm{TB} \quad 0.5-0.9 \mathrm{mg} / \mathrm{dL}\end{array}$ & 8.1 & 0.88 & $89 / 64$ & $46 / 94$ & 15.4 & 0.93 & $82 / 82$ & $90 / 71$ \\
\hline ARFI & 1.27 & 0.84 & $77 / 85$ & $89 / 70$ & 1.65 & 0.89 & $89 / 84$ & $36 / 99$ \\
\hline 2D-SWE & 10.2 & 0.94 & $82 / 93$ & $90 / 88$ & 16.4 & 0.95 & $94 / 91$ & $71 / 99$ \\
\hline MRE & 2.59 & 0.81 & $78 / 78$ & $83 / 83$ & 4.0 & 0.98 & $100 / 92$ & $68 / 100$ \\
\hline
\end{tabular}

AST, aspartate aminotransferase; AUROC, area under receiver operator characteristic curve; ELF, Enhanced liver fibrosis; PGAA, Prothrombin index, gamma glutamyl transferase, Apolipoprotein A1 and $\alpha 2$ macroglobulin; TE, transient elastography; ARFI, acoustic radiation force imaging; 2D-SWE, two dimensional shear wave elastography; MRE, magnetic resonance elastography; PPV, positive predictive value; NPV, negative predictive value; TB, total bilirubin.

to cirrhosis (1). Identification of early stages of ALD is clinically relevant to promote abstinence and halt the fate of progressive ALD (2). This is relevant not only at an individual basis by improving survival, but at a society level by decreasing the enormous cost of ALD in healthcare (4).

\section{Blood-based biomarkers}

Assessment of fibrosis: currently, the most commonly used blood-based biomarkers in ALD patients are ELF and FT with numerous studies evaluating the performance of these tests in liver fibrosis. These tests, along with other frequently used biomarkers in clinical practice are summarized in Table 2.

The PGA index, which includes prothrombin index (PT), GGT and Apolipoprotein A1 was the first blood-based biomarker panel evaluated for detection of cirrhosis among heavy drinkers (67). The addition of $\alpha 2$-macroglobulin to the PGA conformed the PGAA index. PGAA index has been tested in a cohort of 525 alcoholic patients with different histological stages of fibrosis and performed better than PGA in detecting significant fibrosis or cirrhosis with a correct classification in $70 \%$ for PGAA and $65 \%$ for PGA $(\mathrm{P}<0.001)$. In a sub-analysis of asymptomatic patients, PGAA index showed a sensitivity of $89 \%$ and specificity of $79 \%$ for the diagnosis of cirrhosis, using a cut-off of 7 points, suggesting a potential role for the detection of early cirrhosis among heavy drinkers (68). Importantly, when the optimal cutoff value was evaluated based on AUROC, PGAA showed mixed results for significant fibrosis and cirrhosis prediction (Table 2).

Forns index which is composed on 4 routine clinical variables (e.g., age, platelet count, cholesterol levels and GGT) has been developed to predict advanced fibrosis in patients with chronic VHC (69). A few studies have evaluated the performance of Forns index for detecting significant fibrosis $(\geq \mathrm{F} 2)$ and cirrhosis in patients with ALD. In the first study, including 218 ALD patients the AUROC for Forns index was 0.38 and 0.38 for detecting significant fibrosis and cirrhosis, respectively (70). Similarly, in a real-life study including 49 patients with ALD, transient elastography (TE) was significantly better for diagnosing 
advanced fibrosis than Forns index, with corresponding AUROCS of 0.76 and 0.64 (71).

APRI was evaluated in a cohort of 507 patients with ALD finding that values of $>1.5$ had sensitivity and specificity of $13.2 \%$ and $77.6 \%$ for the detection of significant fibrosis and $16.9 \%$ for sensitivity and $86.4 \%$ for specificity for cirrhosis diagnosis at a cut-off of 2 (72). This poor diagnostic performance of APRI was later confirmed in a study comparing TE with non-invasive blood biomarkers. This study included a cohort of 103 ALD patients in where APRI yielded the lowest AUROC at 0.56, while FibroTest/ Fibrosure and PGAA showed a AUROCs of 0.84 and 0.83 respectively (73).

Since both Forns index and APRI were developed for patients with $\mathrm{HCV}$, the potential causes explaining their poor performance in ALD can be due to: (I) the different AST/ALT ratio in HCV patients; (II) decrease in platelets directly related to alcohol consumption and independent of fibrosis stage, and (III) the decrease in GGT values in patients with cirrhosis not being homogeneously reproduced in active alcohol drinkers. For these reasons, APRI and Forns index are not widely used for the noninvasive assessment of fibrosis in patients with ALD.

ELF score showed good performance for prediction of histological stage of liver fibrosis (74) in a meta-analysis including patients with various etiologies of liver disease, and a recent prospective European study including almost 300 ALD patients from primary and secondary health care centers found that ELF and FT had similar diagnostic performance for advanced fibrosis detection in patients with ALD with an AUROC of 0.92 for ELF and an AUROC of 0.90 for FT. Importantly, this study did not find any differences in the diagnostic accuracy of the scoring systems between primary and secondary care centers and demonstrated that ELF values below 10.5 and FibroTest values below 0.58 had NPV for advanced liver fibrosis of $94 \%$ and $90 \%$, respectively in their overall cohort (75).

\section{Imaging-based techniques}

Assessment of fibrosis: as for NAFLD, we will restrict our analysis for the three most frequently studied (Table 2).

$T E / L S M$ accuracy was recently reported in an individual patient data meta-analysis on 1026 ALD patients. In this study, total bilirubin and AST showed a significant influence on LSM, with higher concentrations of these parameters associated with higher LSM values $(\mathrm{P}<0.0001)$, and with significantly higher cut-off values for diagnosis of all fibrosis stages except for $\mathrm{F} \geq 1$. Table 2 summarizes the specific LSM cut-offs adjusted according to concentrations of AST and bilirubin (76). This study is in line with previously informed data on the influence of inflammatory parameters (e.g., AST) on LSM of patients with ALD $(77,78)$. In the study by Mueller et al., excluding patients with AST $>100 \mathrm{U} / \mathrm{L}$ at the time of LSM, significantly improved the AUROC for cirrhosis detection from 0.921 to 0.945 , while specificity increased from $80 \%$ to $90 \%$ at a sensitivity of $96 \%$. Additionally, a similar AUROC could be obtained for F0-2 fibrosis stage if LSM was restricted to patients with AST $<50$ U/L (77). For these reasons, an actual laboratory testing for correct interpretation of LSM may be required in patients with ALD.

SWE has also been studied in patients with ALD, although data are scarce. In one study, ARFI showed a relatively good performance predicting significant fibrosis and cirrhosis with an AUROC of 0.84 and 0.89 , respectively. Of note, in this study the authors found higher values of ALT levels where associated with increased LSM suggesting a positive influence of inflammation on ARFI values (79). Another recent prospective study including 199 heavy drinkers evaluated the predictive performance of 2D-SWE for F3-4 and compared it to TE. Both 2D-SWE and TE identified subjects with significant fibrosis and cirrhosis with high accuracy with no difference in diagnostic accuracy between techniques (80) (Table 2). Finally, although several studies have been done to directly compare the performance of TE with ARFI or 2D-SWE in different etiologies of liver disease, no robust data are available on ALD.

MRE performance to detect fibrosis in ALD is unknown. One study compared the diagnostic accuracy of MRE and Fibrometer ${ }^{\circledR}$ in 90 patients with ALD, however, liver biopsy was not used as the standard of reference and rather fibrosis staging was defined by TE. Such design does not allow us to provide a fair assessment of the accuracy of MRE in ALD (81).

Assessment of steatosis: similar to NAFLD, the major drawback of this technique is the operator dependability affecting diagnostic accuracy. Yet, liver fat can be fairly identified by standard ultrasonography with sensitivities and specificities for all grades of steatosis in the order of $60-94 \%$ and $88-95 \%$, respectively, with higher accuracy when fat represents $>30 \%$ of liver (82).

$C A P$ accuracy in ALD was recently reported as part of a European multicenter prospective study including almost 600 patients. CAP showed a fairly good diagnostic accuracy for mild, moderate and severe steatosis with an AUROC of $0.77,0.78$ and 0.82 , respectively, and it 


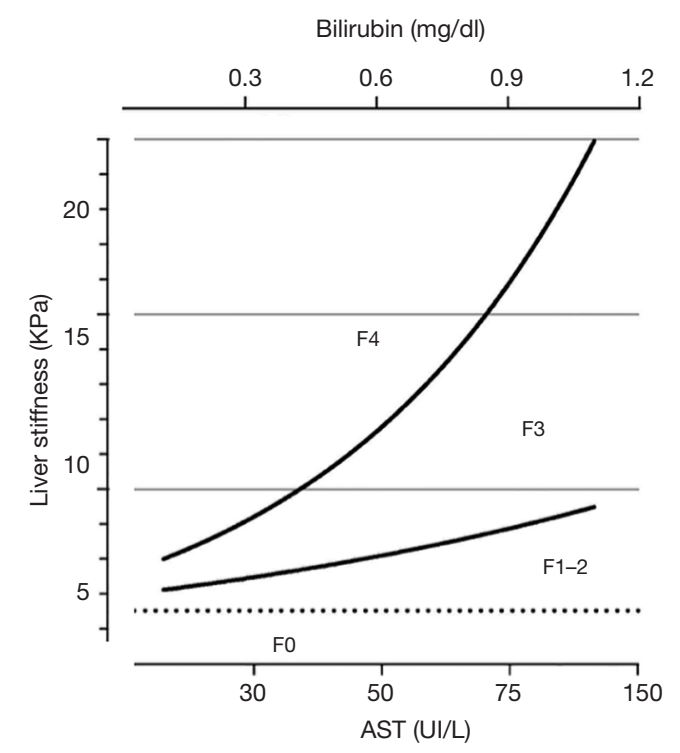

Figure 6 Influence of AST elevation and Bilirubin concentration on liver stiffness measurement in ALD patients. ALD, alcoholrelated liver disease; AST, aspartate aminotransferase.

outperformed regular ultrasound. A CAP above $290 \mathrm{~dB} / \mathrm{m}$ ruled in any steatosis with $88 \%$ specificity and $92 \% \mathrm{PPV}$, while CAP below $220 \mathrm{~dB} / \mathrm{m}$ ruled out steatosis with $90 \%$ sensitivity, but $62 \%$ NPV (83). Similar to NAFLD, CAP technology is a valuable tool for ALD patients particularly for patients with moderate and severe steatosis, although cutoffs values might need to be better optimized to rule in/rule out early ALD.

PDFF accuracy to quantify steatosis in ALD has not been specifically tested. It is likely, however, that its accuracy will not be different to that observed in patients with NAFLD. Although this technique is gaining popularity, appropriate platforms are not widely available, and the cost is a major drawback for population-level ALD screening.

\section{Impact of alcoholic steatohepatitis and abstinence on NIALD}

Histological features of alcoholic steatohepatitis influence LSM values, and coexisting steatohepatitis markedly increases LSM in patients with ALD independently of fibrosis stage (77). In a recent meta-analysis, histological features of steatohepatitis were analyzed in 790 asymptomatic patients (i.e., non-severe form). LSM was significantly elevated in patients with histologically confirmed asymptomatic alcoholic hepatitis compared to patients without histologically confirmed alcoholic hepatitis (median LSM 21 vs. $8.0 \mathrm{kPa}$, respectively; $\mathrm{P}<0.0001$ ), and both AST and bilirubin concentrations affected LSM results reflecting histological alcoholic hepatitis (76). It is well known that cessation of alcohol consumption improves inflammatory liver lesions in patients with ALD. Alcohol consumption or cessation influences LSM values. In this regard, the dynamics of LSM in patients undergoing alcohol detoxification showed a significant decrease in LSM after a median of 5.3 days of complete alcohol abstinence (mean decrease in LSM $3.5 \mathrm{kPa}$, maximum decrease $26.3 \mathrm{kPa} ; \mathrm{P}<0.001$ ), correlating with a significant decrease in AST and bilirubin levels (77). A similar study found a decrease in the mean LSM value at 1 week of detoxification, with $57 \%$ of patients having a significant decrease (>20\% variation) in LSM. Both studies support that LSM variations may be useful to assess alcohol abuse and monitor alcohol relapse in ALD.

In a recent prospective study evaluating CAP in alcoholic steatosis, $75 \%$ of the subgroup of patients with BMI $<30 \mathrm{~kg} / \mathrm{m}^{2}$ admitted for alcohol detoxification showed a rapid decrease in CAP (mean decrease: $32 \pm 47 \mathrm{~dB} / \mathrm{m}$ ) after short-term alcohol withdrawal. In contrast, obese alcohol-overusing patients were more likely to maintain higher CAP values than their non-obese counterparts, irrespective of drinking (83). In summary, kinetics of LSM and CAP may be useful methods for monitoring alcohol abuse and relapse in patients with ALD. However, raised AST and bilirubin concentrations are associated with higher LSM values and should be taken into account when evaluating patients with alcoholic steatohepatitis (Figure 6). In these cases, for the better performance of LSM in fibrosis detection we recommend waiting until AST levels decrease to below $100 \mathrm{UI} / \mathrm{L}$.

\section{Prognosticating ALD outcomes}

While histological fibrosis stage has been showed to be the strongest predictor of outcomes in early, compensated and decompensated ALD, the predictive accuracy of NIALD remains to be established. In fact, the only head-to-head comparison of the prognostic performance of NIALD was recently reported in abstract form. In this study, FibroTest, ELF, LSM and 2D-SWE were compared with histological fibrosis stage in a prospective longitudinal study including 250 patients with history of excessive drinking and no evidence of decompensated cirrhosis. Patients were followed for a median of 32 months and liver-related events were recorded. In this cohort, FibroTest, ELF, TE, and 2D-SWE 
predicted liver related outcomes and death with AUROC of $0.80 / 0.74,0.84 / 0.80,0.86 / 0.780 .85 / 0.75$, respectively, and was comparable to fibrosis stage at $0.79 / 0.79$, respectively. ELF and TE were the strongest predictors of death and outcomes when compared with histological grade of liver fibrosis. Although promising, further data are needed to make conclusions regarding the precise usefulness of NIALD for prognosticating ALD clinically-relevant outcomes.

\section{A practical approach on the use of NIALD for patients with ALD}

Before patients with ALD undergo laboratory or imaging evaluations, a clinical diagnosis of alcohol use disorder needs to be pursued. This is a major challenge since the majority of patients with ALD often appear asymptomatic until they develop advanced disease, and heavy alcohol consumption is difficult to detect, relying on self-report. Brief questionnaires aimed at detecting at-risk individuals including all patients with liver disease should be routinely performed. The 4-item CAGE questionnaire is the briefest effective screening test for lifetime alcohol abuse/ dependence, although the AUDIT score better detects heavy and active drinking (proposed cutoff for AUDIT $>8$ points) (84). In patients with alcohol use disorder, fatty liver is the initial ALD lesion raised by ultrasound or other forms of imaging. Alcoholic fatty liver develops in the majority of heavy drinkers and may be seen within 2 weeks of heavy and regular alcohol ingestion, but it resolves rapidly following complete abstinence (5). After establishing the diagnosis of alcohol use disorder, we recommend testing for fibrosis and steatosis with TE (LSM/CAP) given that this technique identifies twice as many patients with advanced fibrosis/cirrhosis than conventional ultrasound. This is especially important in ALD patients since about half of the patients with apparently early disease may already have significant fibrosis or cirrhosis on liver biopsy when evaluated. Clinicians, however, should have a high suspicion to diagnose ALD in patients with significant fibrosis without steatosis given the rapid disappearance of the latter after a short period of abstinence. If LSM is elevated and patients have AST $>100 \mathrm{U} / \mathrm{mL}$, alcohol abstinence for at least 2 weeks is recommended followed by a second LSM. In patients with $\mathrm{LS}>30 \mathrm{kPa}$, the diagnosis of cirrhosis is usually confirmed, irrespective of steatohepatitis as suggested by elevated AST levels. At such $\mathrm{kPa}$ levels, the development of ascites is very likely. In the absence of raised AST levels, the cut-off values normally used for fibrosis stratification are as follows: F0 $<6 \mathrm{kPa}, \mathrm{F} 2>8 \mathrm{kPa}$, $\mathrm{F} 3>$ and cirrhosis $>12.5 \mathrm{kPa}$. Patients with $>20 \mathrm{kPa}$ deserve screening for esophageal varices and HCC, similar to what was described in the NAFLD section. A proposed algorithm for this stratification and potential therapeutic maneuvers is provided in Figure 7.

\section{Weighing the effects of NAFLD and ALD in the pool of liver donors with NIALD}

Allograft function following liver transplantation depends at least partially on the quality of the transplanted organ, with higher degrees of steatosis further compromising immediate patient recovery. In deceased donation, macrovesicular steatosis fractions above $50-60 \%$ of parenchyma would render the allograft non-transplantable (i.e., discarded organ) under most circumstances, whereas fractions between $30-60 \%$ are acceptable for recipients without severe liver dysfunction. However, among living liver donors, a more stringent limit of $10 \%$ of steatosis is used across most transplant centers in order to maximize donor safety. The growing prevalence of NAFLD and ALD is thus affecting the pool of eligible liver donors, further impacting global liver health. Interestingly, there is a geographic distribution as up to $40 \%$ of donors in the Western world are expected to have macrovesicular steatosis $\geq 30 \%(85,86)$.

Although ultrasonography or non-contrasted computed tomography (liver-to-spleen attenuation $>1.1$ ) are reliable in ruling out moderate to severe steatosis, these techniques are not sensitive enough to identify mild steatosis in the setting of living donation $(87,88)$. CAP is an attractive method to investigate steatosis among liver donors given its high NPV, however, it performs better in the identification of moderate (AUROC 0.88; with a NPV of $98 \%$ at a cut-off value of $276 \mathrm{~dB} / \mathrm{m}$ ) than mild (AUROC 0.78 ) steatosis, which is why it is potentially a more attractive method for deceased rather than living donation (89). Performance of TE, however, can be improved when CAP and LSM are combined (90), or when computed tomography liver-to-spleen attenuation is considered as well (91). The benefits of CAP plus LSM include the possibility of performing point-of-care analysis including evaluation of inpatients (ICU or the operating room) and peripheral clinics with the use of a portable device. However, as recently shown, cut-off values for LSM and CAP need to be tailored for each clinical scenario including the ICU-treated deceased donor $v s$. outpatient living donor, along with donor clinical characteristics (e.g., 


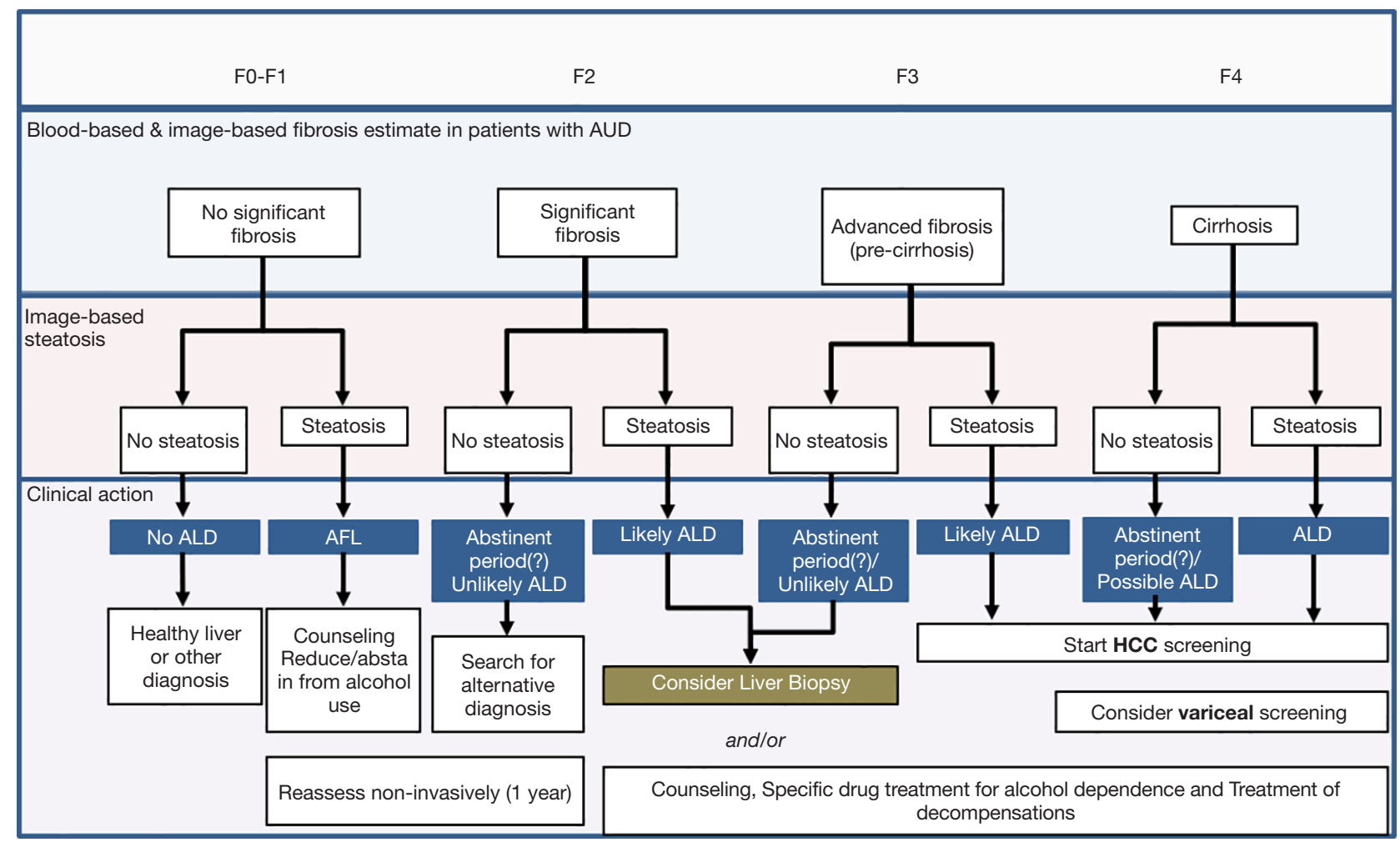

Figure 7 Proposed algorithm for fibrosis stratification, potential screening, and management in patients with alcohol-related liver disease (ALD). AFL, alcoholic fatty liver.

obesity and diabetes) $(49,92)$.

MRI-based methods are the most reliable to identify steatosis, particularly at early stages, and thus more suitable to maximize safety in living donor liver transplantation $(93,94)$. A study including 362 living donors in Korea found MRI-PDFF to be elevated among patients with biopsy-proven NAFLD (significant steatosis irrespective of inflammation or NASH features), when compared to those with normal parenchyma or with any degree of fibrosis $(\mathrm{F} \geq 1)$, and a good correlation with macrovesicular steatosis $(\mathrm{r}=0.77, \mathrm{P}<0.001)$. The AUROC for detection of significant steatosis $(\geq 10 \%)$ was 0.93 and using a cut-off value $>5.8 \%$ PDFF showed a PPV of $48 \%$ and NPV of $98 \%$. Not surprisingly, this study also found MRE to be quite useful in ruling out fibrosis (AUROC 0.85) with an NPV of 99\% (cut-off value $>1.94 \mathrm{kPa}$ ). Similar results were reported for MRI-PDFF and MR spectroscopy-PDFF in the Western population with a limited sample of 32 biopsied patients (94). Although MRI-PDFF and MRE remain the most accurate NIALD tools to assess liver donors, these techniques are not widely available, are more time consuming and expensive, and require a more skillful execution when compared to other NIALD.

\section{Conclusions}

NAFLD and ALD are becoming the leading causes of chronic liver disease worldwide and they covey an important health and socio-economical burden. Accumulated evidence on the use of NIALD in patients with NAFLD and ALD allow now both early detection and prognostication. When approaching patients with presumed NAFLD, we suggest using NFS or FIB-4 and LSM/CAP or MRE/PDFF to stage patients, given their demonstrated accuracy both in cross-sectional and longitudinal studies. NFS and FIB-4 are convenient as they can be calculated from routine blood work, and LSM/CAP allows point-of-care clinical decisions. Although MRE/PDFF likely constitutes the most accurate tools to quantify fibrosis and steatosis, they are not widely available yet. Research on ALD is less abundant than that on NAFLD, however, ELF and FibroTest have been proven useful in assessing fibrosis staging, and we recommend 
testing for fibrosis and steatosis with LSM/CAP as well. LSM/CAP can also be used to identify early liver damage from alcohol abuse and to monitor relapse among alcoholics. LSM/CAP and MRI/MRE show a promising role in evaluating steatosis and fibrosis among deceased and living liver donors. Finally, when using NIALD, clinicians should not forget their limitations and weigh in the effect of some clinical variables (e.g., fasting, BMI, diabetes, hemolysis, etc.) for the proper interpretation of results.

\section{Acknowledgments}

None.

\section{Footnote}

Conflicts of Interest: The authors have no conflicts of interest to declare.

Ethical Statement: The authors are accountable for all aspects of the work in ensuring that questions related to the accuracy or integrity of any part of the work are appropriately investigated and resolved.

\section{References}

1. Parikh ND, Marrero WJ, Wang J, et al. Projected increase in obesity and non-alcoholic-steatohepatitis-related liver transplantation waitlist additions in the United States. Hepatology 2019;70:487-95.

2. Cholankeril G, Wong RJ, Hu M, et al. Liver Transplantation for Nonalcoholic Steatohepatitis in the US: Temporal Trends and Outcomes. Dig Dis Sci 2017;62:2915-22.

3. Pais R, Barritt ASt, Calmus Y, et al. NAFLD and liver transplantation: Current burden and expected challenges. J Hepatol 2016;65:1245-57.

4. Barritt ASt, Jiang Y, Schmidt M, et al. Charges for Alcoholic Cirrhosis Exceed All Other Etiologies of Cirrhosis Combined: A National and State Inpatient Survey Analysis. Dig Dis Sci 2019;64:1460-9.

5. Singal AK, Bataller R, Ahn J, et al. ACG Clinical Guideline: Alcoholic Liver Disease. Am J Gastroenterol 2018;113:175-94.

6. Ajmera V, Belt P, Wilson LA, et al. Among Patients With Nonalcoholic Fatty Liver Disease, Modest Alcohol Use Is Associated With Less Improvement in Histologic Steatosis and Steatohepatitis. Clin Gastroenterol Hepatol 2018;16:1511-1520.e5.

7. Younossi ZM, Stepanova M, Ong J, et al. Effects of Alcohol Consumption and Metabolic Syndrome on Mortality in Patients With Nonalcoholic and AlcoholRelated Fatty Liver Disease. Clin Gastroenterol Hepatol 2019;17:1625-1633.e1.

8. Rockey DC, Caldwell SH, Goodman ZD, et al. Liver biopsy. Hepatology 2009;49:1017-44.

9. Estes C, Anstee QM, Arias-Loste MT, et al. Modeling NAFLD disease burden in China, France, Germany, Italy, Japan, Spain, United Kingdom, and United States for the period 2016-2030. J Hepatol 2018;69:896-904.

10. Duarte-Rojo A, Altamirano JT, Feld JJ. Noninvasive markers of fibrosis: key concepts for improving accuracy in daily clinical practice. Ann Hepatol 2012;11:426-39.

11. Castera L, Friedrich-Rust M, Loomba R. Noninvasive Assessment of Liver Disease in Patients With Nonalcoholic Fatty Liver Disease. Gastroenterology 2019;156:1264-1281.e4.

12. Chalasani N, Younossi Z, Lavine JE, et al. The diagnosis and management of nonalcoholic fatty liver disease: Practice guidance from the American Association for the Study of Liver Diseases. Hepatology 2018;67:328-57.

13. Kleiner DE, Brunt EM, Van Natta M, et al. Design and validation of a histological scoring system for nonalcoholic fatty liver disease. Hepatology 2005;41:1313-21.

14. Brunt EM, Janney CG, Di Bisceglie AM, et al. Nonalcoholic steatohepatitis: a proposal for grading and staging the histological lesions. Am J Gastroenterol 1999;94:2467-74.

15. Altamirano J, Miquel R, Katoonizadeh A, et al. A histologic scoring system for prognosis of patients with alcoholic hepatitis. Gastroenterology 2014;146:1231-9. e1-6.

16. Angulo P, Kleiner DE, Dam-Larsen S, et al. Liver Fibrosis, but No Other Histologic Features, Is Associated With Long-term Outcomes of Patients With Nonalcoholic Fatty Liver Disease. Gastroenterology 2015;149:389-97.e10.

17. Vilar-Gomez E, Calzadilla-Bertot L, Wai-Sun Wong V, et al. Fibrosis Severity as a Determinant of Cause-Specific Mortality in Patients With Advanced Nonalcoholic Fatty Liver Disease: A Multi-National Cohort Study. Gastroenterology 2018;155:443-457.e17.

18. Li B, Zhang C, Zhan YT. Nonalcoholic fatty liver disease cirrhosis: a review of its epidemiology, risk factors, clinical presentation, diagnosis, management, and prognosis. Can J Gastroenterol Hepatol 2018;2018:2784537.

19. Rosenberg WM, Voelker M, Thiel R, et al. Serum markers 
detect the presence of liver fibrosis: a cohort study. Gastroenterology 2004;127:1704-13.

20. Younossi ZM, Loomba R, Anstee QM, et al. Diagnostic modalities for nonalcoholic fatty liver disease, nonalcoholic steatohepatitis, and associated fibrosis. Hepatology 2018;68:349-60.

21. Xiao G, Zhu S, Xiao X, et al. Comparison of laboratory tests, ultrasound, or magnetic resonance elastography to detect fibrosis in patients with nonalcoholic fatty liver disease: A meta-analysis. Hepatology 2017;66:1486-501.

22. Jiang W, Huang S, Teng H, et al. Diagnostic accuracy of point shear wave elastography and transient elastography for staging hepatic fibrosis in patients with nonalcoholic fatty liver disease: a meta-analysis. BMJ Open 2018;8:e021787.

23. Angulo P, Hui JM, Marchesini G, et al. The NAFLD fibrosis score: a noninvasive system that identifies liver fibrosis in patients with NAFLD. Hepatology 2007;45:846-54.

24. Massoud OI, Zein NN. The Effect of Transjugular Intrahepatic Portosystemic Shunt on Platelet Counts in Patients With Liver Cirrhosis. Gastroenterol Hepatol (N Y) 2017;13:286-91.

25. Sterling RK, Lissen E, Clumeck N, et al. Development of a simple noninvasive index to predict significant fibrosis in patients with $\mathrm{HIV} / \mathrm{HCV}$ coinfection. Hepatology 2006;43:1317-25.

26. Wai CT, Greenson JK, Fontana RJ, et al. A simple noninvasive index can predict both significant fibrosis and cirrhosis in patients with chronic hepatitis C. Hepatology 2003;38:518-26.

27. Harrison SA, Oliver D, Arnold HL, et al. Development and validation of a simple NAFLD clinical scoring system for identifying patients without advanced disease. Gut 2008;57:1441-7.

28. Ratziu V, Massard J, Charlotte F, et al. Diagnostic value of biochemical markers (FibroTest-FibroSURE) for the prediction of liver fibrosis in patients with non-alcoholic fatty liver disease. BMC Gastroenterol 2006;6:6.

29. Guha IN, Parkes J, Roderick P, et al. Noninvasive markers of fibrosis in nonalcoholic fatty liver disease: Validating the European Liver Fibrosis Panel and exploring simple markers. Hepatology 2008;47:455-60.

30. Su Y, Gu H, Weng D, et al. Association of serum levels of laminin, type IV collagen, procollagen III N-terminal peptide, and hyaluronic acid with the progression of interstitial lung disease. Medicine (Baltimore) 2017;96:e6617.
31. Hagström H, Nasr P, Ekstedt M, et al. Accuracy of Noninvasive Scoring Systems in Assessing Risk of Death and Liver-Related Endpoints in Patients With Nonalcoholic Fatty Liver Disease. Clin Gastroenterol Hepatol 2019;17:1148-1156.e4.

32. Stern C, Castera L. Non-invasive diagnosis of hepatic steatosis. Hepatol Int 2017;11:70-8.

33. Kotronen A, Peltonen M, Hakkarainen A, et al. Prediction of non-alcoholic fatty liver disease and liver fat using metabolic and genetic factors. Gastroenterology 2009;137:865-72.

34. Fedchuk L, Nascimbeni F, Pais R, et al. Performance and limitations of steatosis biomarkers in patients with nonalcoholic fatty liver disease. Aliment Pharmacol Ther 2014;40:1209-22.

35. European Association for the Study of the L, European Association for the Study of D, European Association for the Study of O. EASL-EASD-EASO Clinical Practice Guidelines for the management of non-alcoholic fatty liver disease. J Hepatol 2016;64:1388-402.

36. Buzzetti E, Hall A, Ekstedt M, et al. Collagen proportionate area is an independent predictor of longterm outcome in patients with non-alcoholic fatty liver disease. Aliment Pharmacol Ther 2019;49:1214-22.

37. Vuppalanchi R, Weber R, Russell S, et al. Is Fasting Necessary for Individuals With Nonalcoholic Fatty Liver Disease to Undergo Vibration-Controlled Transient Elastography? Am J Gastroenterol 2019;114:995-7.

38. Barr RG, Ferraioli G, Palmeri ML, et al. Elastography Assessment of Liver Fibrosis: Society of Radiologists in Ultrasound Consensus Conference Statement. Radiology 2015;276:845-61.

39. Myers RP, Pomier-Layrargues G, Kirsch R, et al. Feasibility and diagnostic performance of the FibroScan $\mathrm{XL}$ probe for liver stiffness measurement in overweight and obese patients. Hepatology 2012;55:199-208.

40. Kennedy P, Wagner M, Castera L, et al. Quantitative Elastography Methods in Liver Disease: Current Evidence and Future Directions. Radiology 2018;286:738-63.

41. Liu H, Fu J, Hong R, et al. Acoustic Radiation Force Impulse Elastography for the Non-Invasive Evaluation of Hepatic Fibrosis in Non-Alcoholic Fatty Liver Disease Patients: A Systematic Review \& Meta-Analysis. PLoS One 2015;10:e0127782.

42. Herrmann E, de Ledinghen V, Cassinotto C, et al. Assessment of biopsy-proven liver fibrosis by twodimensional shear wave elastography: An individual patient data-based meta-analysis. Hepatology 2018;67:260-72. 
43. Loomba R, Wolfson T, Ang B, et al. Magnetic resonance elastography predicts advanced fibrosis in patients with nonalcoholic fatty liver disease: a prospective study. Hepatology 2014;60:1920-8.

44. Singh S, Venkatesh SK, Loomba R, et al. Magnetic resonance elastography for staging liver fibrosis in non-alcoholic fatty liver disease: a diagnostic accuracy systematic review and individual participant data pooled analysis. Eur Radiol 2016;26:1431-40.

45. Bril F, Ortiz-Lopez C, Lomonaco R, et al. Clinical value of liver ultrasound for the diagnosis of nonalcoholic fatty liver disease in overweight and obese patients. Liver Int 2015;35:2139-46.

46. Paige JS, Bernstein GS, Heba E, et al. A Pilot Comparative Study of Quantitative Ultrasound, Conventional Ultrasound, and MRI for Predicting Histology-Determined Steatosis Grade in Adult Nonalcoholic Fatty Liver Disease. AJR Am J Roentgenol 2017;208:W168-W177.

47. de Moura Almeida A, Cotrim HP, Barbosa DB, et al. Fatty liver disease in severe obese patients: diagnostic value of abdominal ultrasound. World J Gastroenterol 2008;14:1415-8.

48. Sasso M, Miette V, Sandrin L, et al. The controlled attenuation parameter (CAP): a novel tool for the noninvasive evaluation of steatosis using Fibroscan. Clin Res Hepatol Gastroenterol 2012;36:13-20.

49. Karlas T, Petroff D, Sasso M, et al. Individual patient data meta-analysis of controlled attenuation parameter (CAP) technology for assessing steatosis. J Hepatol 2017;66:1022-30.

50. Kramer H, Pickhardt PJ, Kliewer MA, et al. Accuracy of Liver Fat Quantification With Advanced CT, MRI, and Ultrasound Techniques: Prospective Comparison With MR Spectroscopy. AJR Am J Roentgenol 2017;208:92-100.

51. Ajmera V, Park CC, Caussy C, et al. Magnetic Resonance Imaging Proton Density Fat Fraction Associates With Progression of Fibrosis in Patients With Nonalcoholic Fatty Liver Disease. Gastroenterology 2018;155:307-310.e2.

52. Park CC, Nguyen P, Hernandez C, et al. Magnetic Resonance Elastography vs Transient Elastography in Detection of Fibrosis and Noninvasive Measurement of Steatosis in Patients With Biopsy-Proven Nonalcoholic Fatty Liver Disease. Gastroenterology 2017;152:598-607.e2.

53. Caussy C, Reeder SB, Sirlin CB, et al. Noninvasive, Quantitative Assessment of Liver Fat by MRI-PDFF as an Endpoint in NASH Trials. Hepatology 2018;68:763-72.

54. Targher G, Byrne CD, Lonardo A, et al. Non-alcoholic fatty liver disease and risk of incident cardiovascular disease: A meta-analysis. J Hepatol 2016;65:589-600.

55. Angulo P, Bugianesi E, Bjornsson ES, et al. Simple noninvasive systems predict long-term outcomes of patients with nonalcoholic fatty liver disease. Gastroenterology 2013;145:782-9.e4.

56. Boursier J, Vergniol J, Guillet A, et al. Diagnostic accuracy and prognostic significance of blood fibrosis tests and liver stiffness measurement by FibroScan in non-alcoholic fatty liver disease. J Hepatol 2016;65:570-8.

57. Lombardi R, Petta S, Pisano G, et al. FibroScan Detects Cardiovascular Damage in Patients With NAFLD. Clin Gastroenterol Hepatol 2018. [Epub ahead of print].

58. Mangla N, Ajmera VH, Caussy C, et al. Liver Stiffness Severity is Associated With Increased Cardiovascular Risk in Patients With Type 2 Diabetes. Clin Gastroenterol Hepatol 2019. [Epub ahead of print].

59. Petta S, Wong VW, Camma C, et al. Serial combination of non-invasive tools improves the diagnostic accuracy of severe liver fibrosis in patients with NAFLD. Aliment Pharmacol Ther 2017;46:617-27.

60. Feldstein AE, Wieckowska A, Lopez AR, et al. Cytokeratin-18 fragment levels as noninvasive biomarkers for nonalcoholic steatohepatitis: a multicenter validation study. Hepatology 2009;50:1072-8.

61. Siddiqui MS, Yamada G, Vuppalanchi R, et al. Diagnostic Accuracy of Noninvasive Fibrosis Models to Detect Change in Fibrosis Stage. Clin Gastroenterol Hepatol 2019;17:1877-1885.e5.

62. Vilar-Gomez E, Calzadilla-Bertot L, Friedman SL, et al. Serum biomarkers can predict a change in liver fibrosis 1 year after lifestyle intervention for biopsy-proven NASH. Liver Int 2017;37:1887-96.

63. Addepally NS, George N, Martinez-Macias R, et al. Hemoglobin A1c Has Suboptimal Performance to Diagnose and Monitor Diabetes Mellitus in Patients with Cirrhosis. Dig Dis Sci 2018;63:3498-508.

64. Jayakumar S, Middleton MS, Lawitz EJ, et al. Longitudinal correlations between MRE, MRI-PDFF, and liver histology in patients with non-alcoholic steatohepatitis: Analysis of data from a phase II trial of selonsertib. J Hepatol 2019;70:133-41.

65. Loomba R, Kayali Z, Noureddin M, et al. GS-0976 Reduces Hepatic Steatosis and Fibrosis Markers in Patients With Nonalcoholic Fatty Liver Disease. Gastroenterology 2018;155:1463-1473.e6.

66. Shimizu M, Suzuki K, Kato K, et al. Evaluation of the effects of dapagliflozin, a sodium-glucose co-transporter-2 
inhibitor, on hepatic steatosis and fibrosis using transient elastography in patients with type 2 diabetes and nonalcoholic fatty liver disease. Diabetes Obes Metab 2019;21:285-92.

67. Poynard T, Aubert A, Bedossa P, et al. A simple biological index for detection of alcoholic liver disease in drinkers. Gastroenterology 1991;100:1397-402.

68. Naveau S, Essoh BM, Ghinoiu M, et al. Comparison of Fibrotest and PGAA for the diagnosis of fibrosis stage in patients with alcoholic liver disease. Eur J Gastroenterol Hepatol 2014;26:404-11.

69. Forns X, Ampurdanes S, Llovet JM, et al. Identification of chronic hepatitis $\mathrm{C}$ patients without hepatic fibrosis by a simple predictive model. Hepatology 2002;36:986-92.

70. Naveau S, Gaude G, Asnacios A, et al. Diagnostic and prognostic values of noninvasive biomarkers of fibrosis in patients with alcoholic liver disease. Hepatology 2009;49:97-105.

71. Janssens F, de Suray N, Piessevaux H, et al. Can transient elastography replace liver histology for determination of advanced fibrosis in alcoholic patients: a real-life study. J Clin Gastroenterol 2010;44:575-82.

72. Lieber CS, Weiss DG, Morgan TR, et al. Aspartate aminotransferase to platelet ratio index in patients with alcoholic liver fibrosis. Am J Gastroenterol 2006;101:1500-8.

73. Nguyen-Khac E, Chatelain D, Tramier B, et al. Assessment of asymptomatic liver fibrosis in alcoholic patients using fibroscan: prospective comparison with seven non-invasive laboratory tests. Aliment Pharmacol Ther 2008;28:1188-98.

74. Xie Q, Zhou X, Huang P, et al. The performance of enhanced liver fibrosis (ELF) test for the staging of liver fibrosis: a meta-analysis. PLoS One 2014;9:e92772.

75. Thiele M, Madsen BS, Hansen JF, et al. Accuracy of the Enhanced Liver Fibrosis Test vs FibroTest, Elastography, and Indirect Markers in Detection of Advanced Fibrosis in Patients With Alcoholic Liver Disease. Gastroenterology 2018;154:1369-79.

76. Nguyen-Khac E, Thiele M, Voican C, et al. Non-invasive diagnosis of liver fibrosis in patients with alcohol-related liver disease by transient elastography: an individual patient data meta-analysis. Lancet Gastroenterol Hepatol 2018;3:614-25.

77. Mueller S, Millonig G, Sarovska L, et al. Increased liver stiffness in alcoholic liver disease: differentiating fibrosis from steatohepatitis. World J Gastroenterol 2010;16:966-72.

78. Bardou-Jacquet E, Legros L, Soro D, et al. Effect of alcohol consumption on liver stiffness measured by transient elastography. World J Gastroenterol 2013;19:516-22.

79. Zhang D, Li P, Chen M, et al. Non-invasive assessment of liver fibrosis in patients with alcoholic liver disease using acoustic radiation force impulse elastography. Abdom Imaging 2015;40:723-9.

80. Thiele M, Detlefsen S, Sevelsted Moller L, et al. Transient and 2-Dimensional Shear-Wave Elastography Provide Comparable Assessment of Alcoholic Liver Fibrosis and Cirrhosis. Gastroenterology 2016;150:123-33.

81. Bensamoun SF, Leclerc GE, Debernard L, et al. Cutoff values for alcoholic liver fibrosis using magnetic resonance elastography technique. Alcohol Clin Exp Res 2013;37:811-7.

82. Lupşor-Platon M, Stefanescu H, Muresan D, et al. Noninvasive assessment of liver steatosis using ultrasound methods. Med Ultrason 2014;16:236-45.

83. Thiele M, Rausch V, Fluhr G, et al. Controlled attenuation parameter and alcoholic hepatic steatosis: Diagnostic accuracy and role of alcohol detoxification. J Hepatol 2018;68:1025-32.

84. de Oliveira JB, Kerr-Correa F, Lima MC, et al. Validity of alcohol screening instruments in general population gender studies: an analytical review. Curr Drug Abuse Rev 2014;7:59-65.

85. Briceño J, Ciria R, Pleguezuelo $M$, et al. Impact of donor graft steatosis on overall outcome and viral recurrence after liver transplantation for hepatitis $\mathrm{C}$ virus cirrhosis. Liver Transpl 2009; 15:37-48.

86. Lee JY, Kim KM, Lee SG, et al. Prevalence and risk factors of non-alcoholic fatty liver disease in potential living liver donors in Korea: a review of 589 consecutive liver biopsies in a single center. J Hepatol 2007;47:239-44.

87. Jun MJ, Shim JH, Kim SY, et al. Clinical implications of preoperative and intraoperative liver biopsies for evaluating donor steatosis in living related liver transplantation. Liver Transpl 2014;20:437-45.

88. Iwasaki M, Takada Y, Hayashi M, et al. Noninvasive evaluation of graft steatosis in living donor liver transplantation. Transplantation 2004;78:1501-5.

89. Hong YM, Yoon KT, Cho M, et al. Clinical usefulness of controlled attenuation parameter to screen hepatic steatosis for potential donor of living donor liver transplant. Eur J Gastroenterol Hepatol 2017;29:805-10.

90. Mancia C, Loustaud-Ratti V, Carrier P, et al. Controlled Attenuation Parameter and Liver Stiffness Measurements for Steatosis Assessment in the Liver Transplant of Brain 
Dead Donors. Transplantation 2015;99:1619-24.

91. Kim JM, Ha SY, Joh JW, et al. Predicting Hepatic Steatosis in Living Liver Donors via Noninvasive Methods. Medicine (Baltimore) 2016;95:e2718.

92. Duarte-Rojo A, Barone GW, Borja-Cacho D, et al. Liver stiffness measurement, but not controlled attenuation parameter, is increased in deceased liver donors at the time of transplant procurement Am J Transplant 2018;18:406.

doi: $10.21037 / \operatorname{tgh} .2019 .11 .14$

Cite this article as: Altamirano J, Qi Q, Choudhry S, Abdallah M, Singal AK, Humar A, Bataller R, Borhani AA, Duarte-Rojo A. Non-invasive diagnosis: non-alcoholic fatty liver disease and alcoholic liver disease. Transl Gastroenterol Hepatol 2020;5:31.
93. Hwang I, Lee JM, Lee KB, et al. Hepatic steatosis in living liver donor candidates: preoperative assessment by using breath-hold triple-echo MR imaging and $1 \mathrm{H} \mathrm{MR}$ spectroscopy. Radiology 2014;271:730-8.

94. Satkunasingham J, Nik HH, Fischer S, et al. Can negligible hepatic steatosis determined by magnetic resonance imaging-proton density fat fraction obviate the need for liver biopsy in potential liver donors? Liver Transpl 2018;24:470-7. 